\title{
Bayesian Inference over the Stiefel Manifold via the Givens Representation: Appendix
}

Arya A. Pourzanjani*, Richard M. Jiang*, Brian Mitchell*, Paul J. Atzberger ${ }^{\dagger}$ and Linda R. Petzold *

\section{Appendix A: Deriving the Change-of-Measure Term}

We derive the simplified form of the differential form showing that

$$
\bigwedge_{i=1}^{p} \bigwedge_{j=i+1}^{n} G_{j}^{T} d Y_{i}=\prod_{i=1}^{p} \prod_{j=i+1}^{n} \cos ^{j-i-1} \theta_{i j} .
$$

We point out that Khatri and Mardia (1977) provide a similar expression for a slightly different representation, but do not offer a derivation. We start by considering the determinant of the matrix form of the change-of-measure term:

$$
\bigwedge_{i=1}^{p} \bigwedge_{j=i+1}^{n} G_{j}^{T} J_{Y_{i}(\Theta)}(\Theta) d \Theta=\left(\begin{array}{c}
G_{2: n}^{T} J_{Y_{1}(\Theta)}(\Theta) \\
G_{3: n}^{T} J_{Y_{2}(\Theta)}(\Theta) \\
\vdots \\
G_{p: n}^{T} J_{Y_{p}(\Theta)}(\Theta)
\end{array}\right)
$$

For $l=1, \cdots, n$, we define the following shorthand notation

$$
\partial_{i, i+l} Y_{k}:=\frac{\partial}{\partial \theta_{i, i+l}} Y_{k}
$$

and

$$
\partial_{i} Y_{k}:=\left(\begin{array}{llll}
\partial_{i, i+1} Y_{k} & \partial_{i, i+2} Y_{k} & \cdots & \partial_{i n} Y_{k}
\end{array}\right)
$$

In the new notation Equation can be written in the following block matrix form:

$$
\left(\begin{array}{cccc}
G_{2: n}^{T} \partial_{1} Y_{1} & G_{2: n}^{T} \partial_{2} Y_{1} & \cdots & G_{2: n}^{T} \partial_{p} Y_{1} \\
G_{3: n}^{T} \partial_{1} Y_{2} & G_{3: n}^{T} \partial_{2} Y_{2} & \cdots & G_{3: n}^{T} \partial_{p} Y_{2} \\
\vdots & \vdots & \ddots & \vdots \\
G_{p: n}^{T} \partial_{1} Y_{p} & G_{p: n}^{T} \partial_{2} Y_{p} & \cdots & G_{p: n}^{T} \partial_{p} Y_{p}
\end{array}\right) .
$$

*Computer Science Department, University of California, Santa Barbara arya@ucsb.edu

${ }^{\dagger}$ Mathematics Department, University of California, Santa Barbara 
Note that the block matrices above the diagonal are all zero. This can be seen by observing that the rotations in the Givens representation involving elements greater than $i$ will not affect $e_{i}$, i.e. letting $R_{i}:=R_{i, i+1} \cdots R_{i n}$,

$$
Y_{i}=R_{1} R_{2} \cdots R_{p} e_{i}=R_{1} \cdots R_{i} e_{i}
$$

Thus for $j>i, \partial_{j} Y_{i}=0$ and the determinant of Expression A.5 simplifies to the product of the determinant of the matrices on the diagonal i.e. the following expression:

$$
\prod_{i=1}^{p} \operatorname{det}\left(G_{i+1: n}^{T} \partial_{i} Y_{i}\right)
$$

\section{A.1 Simplifying Diagonal Block Terms}

Let $I_{i}$ denote the first $i$ columns of the $n \times n$ identity matrix and let $I_{-i}$ represent the last $n-i$ columns. The term $G_{i+1: n}^{T}$ in expression A.7 can be written as

$$
G_{i+1: n}^{T}=I_{-i}^{T} G^{T}=I_{-i}^{T} R_{p}^{T} \cdots R_{1}^{T} .
$$

To simplify the diagonal block determinant terms in Expression A.7 we take advantage of the following fact

$$
\operatorname{det}\left(G_{i+1: n}^{T} \partial_{i} Y_{i}\right)=\operatorname{det}\left(I_{-i}^{T} R_{p}^{T} \cdots R_{1}^{T}\right)=\operatorname{det}\left(I_{-i}^{T} R_{i}^{T} \cdots R_{1}^{T} \partial_{i} Y_{i}\right)
$$

In other words, the terms $R_{p}^{T} \cdots R_{i+1}^{T}$ have no effect on the determinant. This can be seen by first separating terms so that

$$
\begin{aligned}
\operatorname{det}\left(G_{i+1: n}^{T} \partial_{i} Y_{i}\right) & =\operatorname{det}(\underbrace{I_{-i}^{T}}_{(n-i) \times n} R_{p}^{T} \cdots R_{1}^{T} \underbrace{\partial_{i} Y_{i}}_{n \times(n-i)}) \\
& =\operatorname{det}\left(I_{-i}^{T}\left[R_{p}^{T} \cdots R_{i+1}^{T}\right]\left[R_{i}^{T} \cdots R_{1}^{T} \partial_{i} Y_{i}\right]\right),
\end{aligned}
$$

and then noticing that $R_{i+1} \cdots R_{p}$ affects only the first $i$ columns of the identity matrix so

$$
I_{-i}^{T}\left[R_{p}^{T} \cdots R_{i+1}^{T}\right]=\left(R_{i+1} \cdots R_{p} I_{-i}\right)^{T}=\left(I_{-i}\right)^{T} .
$$

Thus Expression A.7 is equivalent to 


$$
\prod_{i=1}^{p} \operatorname{det}\left(I_{-i}^{T} R_{i}^{T} \cdots R_{1}^{T} \partial_{i} Y_{i}\right)
$$

Now consider the $k, l$ element of the $(n-i) \times(n-i)$ block matrix $I_{-i}^{T} R_{i}^{T} \cdots R_{1}^{T} \partial_{i} Y_{i}$. This can be written as

$$
\begin{aligned}
e_{i+k}^{T} R_{i}^{T} \cdots R_{1}^{T} \partial_{i, i+l} Y_{i} & =e_{i+k}^{T} R_{i}^{T} \cdots R_{1}^{T} \partial_{i, i+l}\left(R_{1} \cdots R_{i} e_{i}\right) \\
& =e_{i+k}^{T} R_{i}^{T} \cdots R_{1}^{T} R_{1} \cdots R_{i-1}\left(\partial_{i, i+l} R_{i} e_{i}\right) \\
& =e_{i+k}^{T} R_{i}^{T}\left(\partial_{i, i+l} R_{i} e_{i}\right) .
\end{aligned}
$$

Since $e_{i+k}^{T} R_{i}^{T} R_{i} e_{i}=0$, taking the derivatives of both sides and applying the product rule yields

$$
\begin{aligned}
& \partial_{i, i+l}\left(e_{i+k}^{T} R_{i}^{T} R_{i} e_{i}\right)=\partial_{i, i+l} 0 \\
\Rightarrow \quad & \left(\partial_{i, i+l} e_{i+k}^{T} R_{i}^{T}\right) R_{i} e_{i}+e_{i+k}^{T} R_{i}^{T}\left(\partial_{i, i+l} R_{i} e_{i}\right)=0 \\
\Rightarrow & e_{i+k}^{T} R_{i}^{T}\left(\partial_{i, i+l} R_{i} e_{i}\right)=-\left(\partial_{i, i+l} e_{i+k}^{T} R_{i}^{T}\right) R_{i} e_{i} .
\end{aligned}
$$

Combining expression A.15 this fact with expression A.14, the expression for the $k, l$ element of $I_{-i}^{T} R_{i}^{T} \cdots R_{1}^{T} \partial_{i} Y_{i}$ becomes $-\left(\partial_{i, i+l} e_{i+k}^{T} R_{i}^{T}\right) R_{i} e_{i}$.

However, note that

$$
e_{i+k}^{T} R_{i}^{T}=e_{i+k}^{T} R_{i n}^{T} \cdots R_{i, i+1}^{T}=e_{i+k}^{T} R_{i, i+k}^{T} \cdots R_{i, i+1}^{T},
$$

and the partial derivative of this expression with respect to $i, i+l$ is zero when $k>l$. Thus it is apparent that $I_{-i}^{T} R_{i}^{T} \cdots R_{1}^{T} \partial_{i} Y_{i}$ contains zeros above the diagonal and that $\operatorname{det}\left(I_{-i}^{T} R_{i}^{T} \cdots R_{1}^{T} \partial_{i} Y_{i}\right)$ is simply the product of the diagonal elements of the matrix.

\section{A.2 Diagonal Elements of the Block Matrices}

To obtain the diagonal terms of the block matrices, we directly compute $-\partial_{i, i+l} e_{i+k}^{T} R_{i}^{T}$ for $l=k, R_{i} e_{i}$, and their inner-product. Defining $D_{i j}:=\partial_{i j} R_{i j}$,

$$
\begin{aligned}
-\partial_{i, i+k} R_{i} e_{i+k} & =-\partial_{i, i+k}\left(R_{i, i+1} \cdots R_{i, i+k} e_{i+k}\right) \\
& =-R_{i, i+1} \cdots R_{i, i+k-1} D_{i, i+k} e_{i+k}
\end{aligned}
$$




$$
=R_{i, i+1} \cdots R_{i, i+k-1}\left(\begin{array}{c}
0 \\
\vdots \\
0 \\
\cos \theta_{i, i+k} \\
0 \\
\vdots \\
0 \\
\sin \theta_{i, i+k} \\
0 \\
\vdots \\
0
\end{array}\right)
$$$$
=R_{i, i+1} \cdots R_{i, i+k-2}\left(\begin{array}{c}
0 \\
\vdots \\
0 \\
\cos \theta_{i, i+k-1} \cos \theta_{i, i+k} \\
0 \\
\vdots \\
\sin \theta_{i, i+k-1} \cos \theta_{i, i+k} \\
\sin \theta_{i, i+k} \\
0 \\
\vdots \\
0
\end{array}\right)
$$

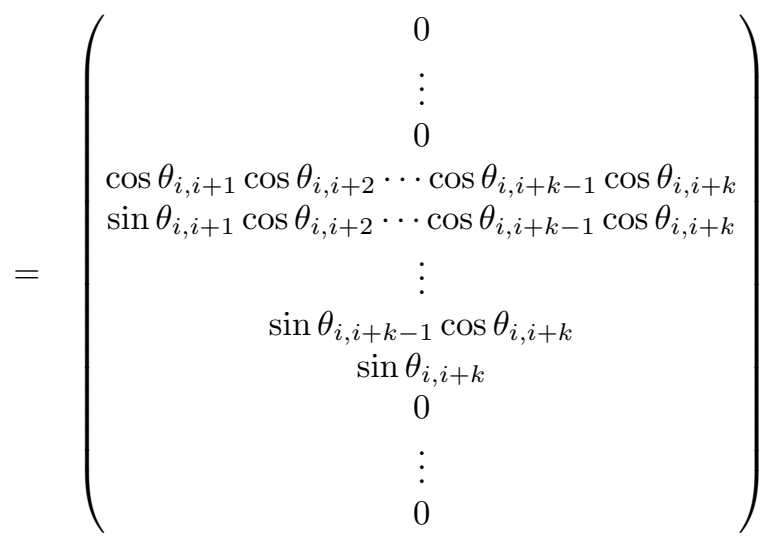

(A.21)

which is zero up to the $i$ th spot. After the $i+k$ th spot,

$$
R_{i} e_{i}=R_{i, i+1} \cdots R_{i n} e_{i}
$$




$$
=\left(\begin{array}{c}
0 \\
\vdots \\
0 \\
\cos \theta_{i, i+1} \cos \theta_{i, i+2} \cdots \cos \theta_{i, n-1} \cos \theta_{i n} \\
\sin \theta_{i, i+1} \cos \theta_{i, i+2} \cdots \cos \theta_{i, n-1} \cos \theta_{i n} \\
\vdots \\
\sin \theta_{i, n-1} \cos \theta_{i n} \\
\sin \theta_{i n}
\end{array}\right) .
$$

Finally, directly computing the inner-product of $-\partial_{i, i+l} e_{i+k}^{T} R_{i}^{T}$ and $R_{i} e_{i}$ yields

$$
\begin{aligned}
-\left(\partial_{i, i+l} e_{i+k}^{T} R_{i}^{T}\right)\left(R_{i} e_{i}\right) & =\cos ^{2} \theta_{i, i+1} \cos ^{2} \theta_{i, i+2} \cdots \cos ^{2} \theta_{i, i+k} \cos \theta_{i, i+k+1} \cdots \cos \theta_{i n} \\
& +\sin ^{2} \theta_{i, i+1} \cos ^{2} \theta_{i, i+2} \cdots \cos ^{2} \theta_{i, i+k} \cos \theta_{i, i+k+1} \cdots \cos \theta_{i n} \\
& +\sin ^{2} \theta_{i, i+2} \cos ^{2} \theta_{i, i+3} \cdots \cos ^{2} \theta_{i, i+k} \cos \theta_{i, i+k+1} \cdots \cos \theta_{i n} \\
& \vdots \\
& +\sin ^{2} \theta_{i, i+k} \cos \theta_{i, i+k+1} \cdots \cos \theta_{i n} \\
& =\cos ^{2} \theta_{i, i+2} \cos ^{2} \theta_{i, i+3} \cdots \cos ^{2} \theta_{i, i+k} \cos \theta_{i, i+k+1} \cdots \cos \theta_{i n} \\
& +\sin ^{2} \theta_{i, i+2} \cos ^{2} \theta_{i, i+3} \cdots \cos ^{2} \theta_{i, i+k} \cos \theta_{i, i+k+1} \cdots \cos \theta_{i n} \\
& \vdots \\
& +\sin ^{2} \theta_{i, i+k} \cos \theta_{i, i+k+1} \cdots \cos \theta_{i n} \\
& =\cdots \\
& =\cos \theta_{i, i+k+1} \cdots \cos \theta_{i n} \\
& =\prod_{k=i+1}^{n} \cos \theta_{i k} .
\end{aligned}
$$

Thus the determinant of the entire block matrix $I_{-i}^{T} R_{i}^{T} \cdots R_{1}^{T} \partial_{i} Y_{i}$ simplifies to

$$
\prod_{k=i+1}^{n}\left(\prod_{j=k+1}^{n} \cos \theta_{i k}\right)=\prod_{j=i+1}^{n} \cos ^{j-i-1} \theta_{i j} .
$$

Combining this with Expression A.13 yields

$$
\prod_{i=1}^{p} \operatorname{det}\left(I_{-i}^{T} R_{i}^{T} \cdots R_{1}^{T} \partial_{i} Y_{i}\right)=\prod_{i=1}^{p} \prod_{j=i+1}^{n} \cos ^{j-i-1} \theta_{i j}
$$




\section{References}

Khatri, C. and Mardia, K. (1977). "The von Mises-Fisher matrix distribution in orientation statistics." Journal of the Royal Statistical Society. Series B (Methodological), $95-106$. 